\title{
Pour en lire plus : Pour une écoformation - Former à et par l'environnement.
}

\section{Tom Berryman}

\section{(2) OpenEdition \\ 12 Journals}

Édition électronique

URL : http://journals.openedition.org/ere/6681

DOI : $10.4000 /$ ere.6681

ISSN : 2561-2271

Éditeur

Centr'ERE

Référence électronique

Tom Berryman, « Pour en lire plus : Pour une écoformation - Former à et par l'environnement. », Éducation relative à l'environnement [En ligne], Volume 3 | 2002, mis en ligne le 20 mars 2002, consulté le 16 avril 2021. URL : http://journals.openedition.org/ere/6681 ; DOI : https://doi.org/10.4000/ere. 6681

Ce document a été généré automatiquement le 16 avril 2021 


\title{
Pour en lire plus : Pour une
} écoformation - Former à et par l'environnement.

\author{
Tom Berryman
}

\section{RÉFÉRENCE}

Pour une écoformation : former à et par l'environnement. Numéro 148 (2001-3) du périodique Éducation permanente. Une collaboration GREF - Ifrée-ORE. 

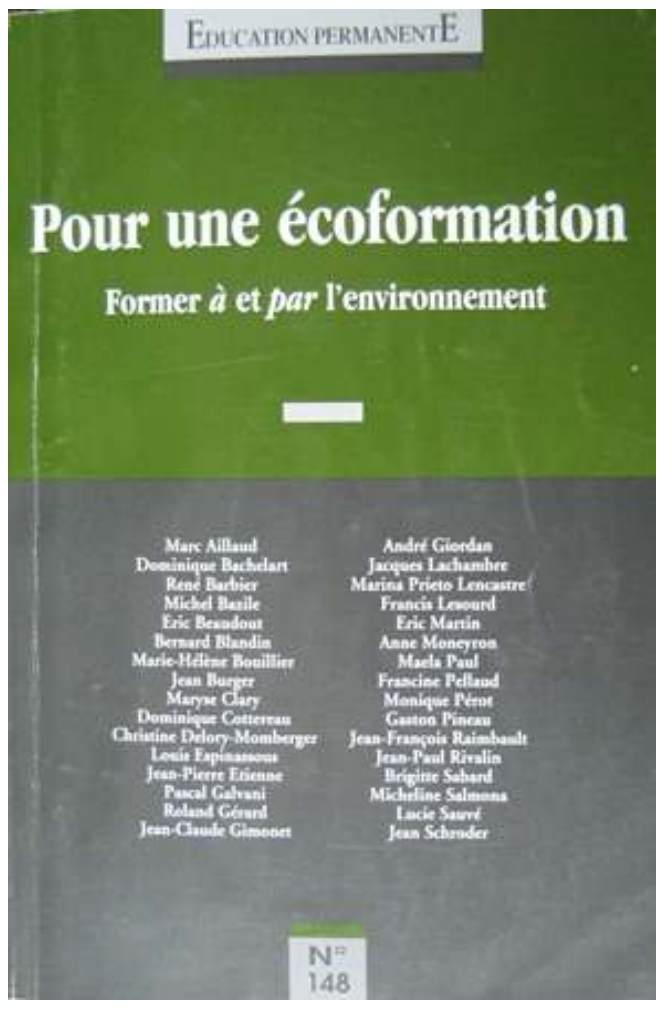

1 Éducation permanente est un trimestriel publié depuis 1969. Voué à la formation continue, il est construit sur la conviction «qu'aujourd'hui comme hier, le besoin d'échanger, de critiquer, d'éclairer la pratique par la recherche demeure aussi fort ». Le numéro 148, annoncé sous le thème "formation et environnement ", a été publié en novembre 2001. Intitulé Pour une écoformation: Former à et par l'environnement, il regroupe, sur près de 300 pages, 26 textes préparés par 32 auteurs. Produit sous la direction de Gaston Pineau, à qui on doit le néologisme écoformation, il est issu d'une collaboration entre le Groupe de recherche sur l'écoformation (GREF) et l'Institut de formation et de recherche en éducation à l'environnement (Ifrée-ORE) en PoitouCharentes.

2 Les articles offrent une vue panoramique sur une mosaïque de perspectives concernées par diverses formes de prise en compte des liens au monde dans la formation des adultes. Les textes sont répartis en quatre sections : 1 ) " paysage théorique contrasté ", 2) "pratiques émergentes diversifiées du champ éducatif», 3) "pratiques émergentes diversifiées du champ professionnel » et enfin, 4) «pour une écoformation, dernière section qui se présente comme une vision synthétique avant tout produite par différents membres du GREF.

L'ensemble des textes rassemblés ouvrent effectivement, chez le lecteur, une sorte d'espace mental où dialoguent des perspectives complémentaires, parfois divergentes, cherchant toutes à intégrer les relations à l'environnement dans la formation. La notion même de formation est ici discutée dans des acceptions des plus restreintes aux plus larges et totalisantes. Pour ceux identifiant leur pratique à l'éducation relative à l'environnement (ERE), ces textes permettent de mieux appréhender et apprécier la notion d'écoformation. Parmi les gens associés au GREF et à l'écoformation, on trouve des textes de René Barbier, Éric Beaudout, Bernard Blandin, Dominique Cottereau, Pascal Galvani, Francis Lesourd, Anne Moneyron et Maela Paul. Chez des gens associés à 
l'Ifrée-ORE ou à l'ERE, on trouve des textes de Jean Burger, Maryse Clary, Roland Gérard, André Giordan, Brigitte Sabard et Lucie Sauvé.

4 Même si l'écoformation et l'ERE sont les tendances lourdes qui infléchissent le discours éducatif des auteurs, on trouve une panoplie d'autres appellations auxquelles certains auteurs se rattachent et qui animent dès lors un panorama conceptuel bigarré : éducation par l'environnement et le développement, éducation à l'environnement et au développement durable, éducation au développement durable, éducation à l'environnement et à la viabilité, pédagogie de l'environnement, animation nature, formations techniques et professionnelles en gestion de l'environnement. Ici comme ailleurs cependant, la durabilité tend à hanter le discours.

Quels sont les constats, observations, hypothèses et questionnements au sortir d'une telle absorption dans ce foisonnement de points de vue?

6 La question de l'environnement, en éducation et en formation, demeure essentiellement portée par un certain nombre de personnes, par quelques rares groupes et de plus rares institutions. Cette question n'a pas fait une entrée très forte dans la formation. Au travers ces écrits, davantage centrés sur la formation des adultes, persiste l'impression d'une certaine marginalité, malgré la richesse et la profondeur des expériences relatées.

7 Comparativement à d'autres périodiques associés à l'ERE, les écrits présentés ici sont davantage des exposés théoriques, des points de vue argumentés et des réflexions sur des pratiques de formation et des visions du monde plutôt que des résultats formels de projets de recherches. Particulièrement au travers des textes portant sur l'écoformation, on est exposé à une exploration plus en profondeur des liens entre la personne et le milieu. Cette ouverture est certes rafraîchissante dans le domaine de l'ERE. Outre une forte influence d'Edgar Morin, via le discours sur la complexité qui est généralisé et répétitif, il est aussi remarquable de constater la place occupée par les travaux de Gaston Bachelard dans les écrits sur l'écoformation. Il semble certes s'agir là d'un trait permettant de distinguer l'écoformation de l'ERE et de l'éducation au développement durable. Une telle ouverture poétique à l'environnement et aux liens avec le milieu de vie, notamment via les quatre éléments que Bachelard emprunte aux Grecs, est porteuse d'un souffle nouveau pour les pratiques de formation. D'ailleurs, les textes portant sur les pratiques éducatives du champ professionnel ont été choisis en fonction des éléments eau, air, terre et feu: navigation de plaisance, aviation civile, agriculture et pompiers. Cette option, si elle donne quelques résultats heureux, nous expose par contre aussi à des textes dont la pertinence est plus douteuse au regard de l'objectif de la publication. Ici, on peut regretter d'avoir sacrifié des écrits possiblement plus pertinents afin de respecter un principe ou un concept - des éléments eau, air, terre et feu.

$8 \mathrm{Au}$ terme de cette lecture, risquons maintenant une hypothèse sur l'articulation entre ERE, éducation au développement durable et écoformation. Le discours dominant sur l'ERE focalise surtout sur l'environnement là-dehors qu'il tend à appréhender par ses problèmes via les sciences et dans une approche systémique. Le discours sur l'éducation au développement durable focalise quant à lui surtout sur les problèmes de développement socio-économique et d'allocations de ressources pour un tel développement qu'il tend à aborder dans une logique à la fois politique et comptable. Enfin, le discours sur l'écoformation focalise surtout sur la construction de l'être-aumonde et sur la personne humaine qu'il tend à approcher en termes de problème 
d'identité de l'être et du rôle de l'environnement dans la constitution et l'évolution de cette identité. Dans cette optique, l'écoformation stimule un renouvellement de l'ERE afin qu'elle se réapproprie une de ses racines bien antérieure à sa légitimation par les organisations internationales (UNESCO) dans les années 1970-1980, soit les rapports au monde et les rapports à la nature dans toute leur amplitude, relations qui ne peuvent et ne devraient pas être réduites à la seule résolution de problèmes socioenvironnementaux, aussi importante que soit cette tentative de les résoudre.

9 En fin de compte, l'ensemble de ces textes constituent une sorte de guide synthétique fort intéressant pour cheminer sur le terrain encore vague des formations qui affirment prendre un appui sur les liens entretenus avec le monde qui nous porte. Présentés côte à côte, ces points de vue différents stimulent les réflexions et devraient enrichir les pratiques.

\section{AUTEUR}

\section{TOM BERRYMAN}

Université du Québec à Montréal 